\title{
INFLUÊNCIA DO ENVELHECIMENTO ACELERADO NAS PROPRIEDADES MECÂNICAS DO PRFV QUANDO IMERSO EM PETRÓLEO
}

\author{
R. A. D. CUNHA ${ }^{*}$, J. K. D. SANTOS, R. D. CUNHA e J. U. L. MENDES \\ Universidade Federal do Rio Grande de Norte \\ ricardoalexc@gmail.com*
}

Submetido 10/12/2016 - Aceito 27/09/2017

DOI: $10.15628 /$ holos.2017.3841

\section{RESUMO}

Este trabalho tem por objetivo fazer uma análise $A$ utilização dos materiais compósitos estar sendo amplamente investigada para a aplicação em formas estruturais, tendo em visto esta utilização o objetivo desse trabalho consiste em fazer uma análise comparativa das propriedades mecânicas de tração uniaxial em compósitos reforçado por fibra de vidro quando os mesmos passaram por uma imersão em petróleo no período de 365 dias e por um envelhecimento acelerado, sob ação simultânea de temperatura e umidade. Após os ensaios mecânicos pode ser feito uma avaliação comparativa do comportamento das propriedades mecânicas como módulo de elasticidade, tensão de ruptura, deformação de ruptura e tensão máxima. Para efeitos de comparação, usou-se o material sem nenhum tipo de ação externa (petróleo ou envelhecimento), ou seja, estado original e o material submetido ao efeito da imersão em petróleo e do envelhecimento acelerado. Ao final, os laminados em estudo, foram submetidos à caracterização final da fratura. Podemos concluir que para o ensaio de tração houve uma queda em torno de $25 \%$ para o material envelhecido e $16 \%$ para o imerso em petróleo, para a tensão máxima, já para o módulo elástico, os percentuais de retenção foram em torno de $28 \%$ e $16 \%$, para o imerso em petróleo e o envelhecido, respectivamente. $\mathrm{Na}$ caracterização da fratura, os principais tipos de danos em materiais compósitos foram observados, como por exemplo, fraturas adesivas, coesivas na fibra e na matriz

PALAVRAS-CHAVE: Propriedades Mecânicas, Materiais Compósitos, Envelhecimento Acelerado, Petróleo.

\section{INFLUENCE OF ACCELERATED AGING IN MECHANICAL PROPERTIES OF GFR IMMERSED IN OIL}

\begin{abstract}
This study aims to analyze the use of composite materials being widely investigated for application in structural forms, having seen this use our aim is to make a comparative analysis of the mechanical properties of uniaxial tensile composite carbon fiber reinforced glass when they passed through an immersion oil in the period of 365 days and an accelerated aging under simultaneous action of temperature and humidity. After the mechanical tests can be made a comparative evaluation of the behavior of mechanical properties such as elastic modulus, rupture stress, break strain, and maximum stress. For comparison purposes, we used the material without any external action (petroleum or aging), that is, original state, and the material subjected
\end{abstract}

to the effect of the immersion oil and accelerated aging. Finally, the laminated study were submitted to the final characterization of the fracture. We can conclude that for the tensile test there was a drop of around $25 \%$ for the aged material and $16 \%$ for the immersed in oil to the maximum voltage, as for elastic modulus, retention rates were around $28 \%$ and $16 \%$ for the immersed in oil and aged, respectively. In characterizing the fracture, the main types of damage in composite materials have been observed, such as fractures adhesive, cohesive in fiber and the matrix.

KEYWORDS: Mechanical Properties, Composite Materials, Accelerated Aging, Oil. 


\section{INTRODUÇÃO}

A necessidade de trabalho no campo industrial requer materiais que devem apresentar propriedades sejam elas mecânicas, térmicas, químicas, entre outras que apresentem bom desempenho estrutural, físico, mecânico, elevada resistência a danos ambientais (umidade, temperatura) e elevado custo/benefício. Diante disso, alguns materiais ditos convencionais estão caindo em desuso visto que não apresentam todas essas vantagens combinadas. Surgem então, os materiais compósitos que possuem características físico/químico-mecânica de larga abrangência e utilização.

Como definição dos materiais compósitos a característica básica é combinar pelo menos duas fases distintas denominadas de matriz e a outra de reforço (MENDONÇA, 2005). O reforço é responsável pelas características mecânicas (rigidez, resistência a ruptura, etc) enquanto que a matriz tem como finalidade principal transferir solicitações mecânicas às fibras e protegê-las do ambiente externo (FELIPE, 1997). Dentre características que podemos citar dos compósitos poliméricos avançados estão: elevada resistência à fadiga e à corrosão, baixa condutividade térmica, isolação acústica e térmica e facilidade de obtenção de geometrias complexas, assegurando-Ihes maior desempenho em serviço (STRONG, 1989 e TAN, 1994).

Os compósitos poliméricos estão sendo empregados nas mais variadas áreas da indústria, como exemplo na fabricação de satélites, aeronaves e helicópteros, implantes ortopédicos e odontológicos biocompatíveis, veículos de fórmula-1, pontes, telescópios, instrumentos musicais e estruturas em geral (LEVY NETO e PARDINI, 2006). Na indústria aeroespacial, em particular, o emprego de compósitos de matriz polimérica abrange desde flanges, leme, carenagens, tanques de combustível, profundo e cone de cauda, entre outros (GAY, 1989, Mc CONNELL, 1990). Na indústria automobilística (fabricação de pára-choque, corroeria de caminhão), náutica (fabricação de barco), química (taques para armazenamento de produtos químicos) entre outras indústrias, (ALBUQUERQUE et al., 1999)

\section{REVISÃO BIBLIOGRÁFICA}

\subsection{Fibras Sintéticas e Aplicações}

Compósitos reforçados com fibras sintéticas têm atraído muita atenção devido às suas melhores propriedades de durabilidade e resistência à umidade. A maior parte desses compósitos é reforçada por fibra de vidro, fibra de carbono, ou kevlar (aramida) aponta Khan, et. all, 2010. Podemos citar como exemplos dessas as fibras de carbono, fibras de Kevlar (aramida) e as fibras de vidro.

Polímeros reforçados com fibras de carbono (PRFC) foram amplamente utilizados na indústria aeroespacial, equipamentos esportivos, e indústrias de automóvel por causa de suas propriedades, tais como peso leve, resistente forte à corrosão e baixa expansão térmica. 0 consumo global de PRFC foi previsto para alcançar aproximadamente 140 mil toneladas (48.700 milhões dólares americanos) em 2020, constituindo um aumento de 46.000 toneladas (16.100 milhões dólares) em 2011. De acordo com previsões da Boeing Company, 8.500 aviões comerciais 
serão aposentados nos próximos 10 anos, cada um representando mais de 20 toneladas de fragmento do PRFC (Huang et al., 2015).

A fibra de Kevlar é uma espécie de fibra de aramida, que é conhecida por sua leveza, baixa dielétrica, baixo coeficiente de expansão térmica (CET) e maior consistência dimensional. Vale salientar que as propriedades-chave no princípio de compósitos, incluindo, propriedades dielétricas e térmicas de processamento não apenas dependente da sua matriz e reforço, mas também de sua adesão interfacial entre ambas. No entanto, compósitos polímeros de fibra de Kevlar geralmente têm pouca adesão interfacial porque a estrutura química inerte e as superfícies lisas das fibras impedem a ligação química e o inter-travamento mecânico de vários substratos levando assim as performances integradas, propriedades dielétricas e especialmente a resistência à umidade (Su et al., 2001).

\subsection{Fibra de Vidro}

Entre todas as fibras sintéticas, as fibras de vidro são as mais usadas, devido ao seu baixo custo e boas propriedades físico-mecânicas. Estas podem se classificar em diferentes tipos: A, B, $C$, E, entre outras. As fibras de vidro são produzidas quando cordões finos ou de outras formulações de vidro são extraídas em vários diâmetros de pequenas fibras à base de sílica em um processamento têxtil. A fibra de vidro mais utilizada é vidro-E, que tem boas propriedades de isolamento e pode manter essas propriedades até $815^{\circ} \mathrm{C}$. Estas são frequentemente empregadas como reforço em matriz polimérica devido à sua maior tensão para a falha, melhor resistência ao impacto, boa vida à fadiga e boa resistência à corrosão nos ambientes mais comuns. A fibra de vidro-E é empregada regularmente em tapetes, isolamento, reforço, absorção sonora, tecidos resistentes ao calor, tecidos resistentes à corrosão, e tecidos de alta resistência (Khan et al., 2010).

Plásticos reforçados com fibra de vidro (PRFV) têm sido utilizados como materiais estruturais já há mais de 50 anos. No início de suas aplicações, muito pouco se sabia sobre mecanismo de fadiga e danos aos PRFV. Esperava-se que a resistência à fadiga será pelo menos tão boas como as dos metais. A propagação de micro fissuras na matriz era esperado para encostarem-se às fibras. Desde então, materiais compósitos com sucesso têm sido aplicados em muitas outras áreas da engenharia e da indústria, não só em planadores e aviões, onde eles permitiram enormes melhorias nas estruturas. Por outro lado, a utilização do PRFV nas industriais de aviões ou turbinas eólicas tem sido lenta, devido ao conservadorismo de muitos designers, que não foram familiarizados com inúmeras diferenças características em comparação com os aços, que devido às propriedades inadequadas dos próprios materiais. (Cerny et al., 2012).

\subsection{Influência da Umidade e da Temperatura}

Os compósitos poliméricos estão cada vez mais presentes no meio industrial, devido sua variedade de condições e combinações específicas para a fabricação de elementos estruturais como perfis, tubulações, escadas entre outros. Com isso, a preferência por esses materiais, que apresentam melhor custo/benefício estão fazendo com que caia a procura pelo emprego dos materiais tradicionais, como materiais metálicos. 
Barjasteh et al. (2009) observou o envelhecimento térmico em plástico reforçado, sendo o compósito híbrido unidirecional reforçado por fibras de vidro/carbono utilizando matriz epóxi, onde submeteu este compósito a uma variação de temperatura entre $180^{\circ} \mathrm{C}$ a $200^{\circ} \mathrm{C}$ por um período de 8736 horas, tendo como objetivo verificar a cinética de oxidação das camadas internas do compósito e as propriedades mecânicas após o período de exposição. Ao término do estudo, foi observado que a camada da superfície que oxidou funcionou como uma camada passiva, impedindo a difusão do oxigênio e protegendo a matriz (epóxi) de uma oxidação mais profunda. O módulo de elasticidade por sua vez, permaneceu parcialmente inalterado pela oxidação térmica após 52 semanas de exposição a essa variação de temperatura. E não foi observada formação de trincas nas amostras envelhecidas termicamente após as exposições no prazo de um ano, aproximadamente 8736 horas.

Shakerin et al. (2010) realizou uma pesquisa, utilizando os seguintes materiais: fibra de vidro-E, jornal reciclado e matriz de polipropileno, para a fabricação de dois compósitos distintos, sendo um hibrido tendo como reforço fibra de vidro/jornal, e outro reforçado com apenas fibra de jornal, ambos utilizando uma matriz de polipropileno, sendo estes compósitos confeccionados pelo processo de prensagem a quente, com isso, os autores visaram fazer uma análise comparativa investigando a absorção de umidade do material, até a sua saturação, sendo estes corpos de provas imersos em água. Diante disso, foi constatado que os compósitos produzidos a partir de jornal reciclado mostraram resultados comparáveis como os feitos de compósito híbrido. É interessante se destacar que a absorção de água e a variação da espessura, devido ao inchaço dos compósitos podem ser reduzidas significativamente com a incorporação de um agente de acoplamento como, por exemplo, o polipropileno maleado.

O trabalho realizado por Cunha et al., (2006) foi analisado o efeito higro-térmico, observando então, o comportamento mecânico à tração longitudinal e transversal do compósito formado por matriz de epóxi e fibras de carbono, onde foi investigado os efeitos da umidade e da temperatura para ambas resistências, diante disso, o referido material foi conduzido a duas câmaras: uma câmara de névoa salínica (salt spray) e outra de climatização, sendo realizados dois procedimentos, no primeiro os corpos de provas (CP's) foram colocados em ambas as câmaras a temperatura ambiente $\left(22^{\circ} \mathrm{C}\right)$ até a saturação do material, no outro procedimento os $\mathrm{CP}^{\prime}$ s foram inseridos nas câmaras, sendo que na de névoa salínica foi utilizada uma temperatura de $46^{\circ} \mathrm{C}$ a uma pressão de $9,81 \mathrm{~N} / \mathrm{cm}^{2}$, e na câmara de climatização a temperatura empregada foi de $80^{\circ} \mathrm{C}$ e $90 \%$ de umidade a pressão atmosférica. Os resultados obtidos mostraram que na temperatura ambiente para tração longitudinal as amostras não perderam sua resistência, porém os compósitos apresentaram uma redução na sua resistência quando submetidos à câmara de climatização. Já nos ensaios de tração transversal observou-se uma redução na resistência em torno de $11 \%$ quando as amostras foram submetidas na câmara de névoa salínica e uma diminuição acentuada cerca de $51 \%$ quando as amostras foram conduzidas à câmara de climatização. Assim sendo, a combinação de altas temperaturas com o condicionamento em câmara de umidade controlada, reduziu as resistências em ambos os casos, devido à degradação da matriz polimérica.

Ciclos térmicos foram analisados por Boccaccini et al., (1998) buscando o comportamento mecânico do composto com a matriz cerâmica reforçada com fibra de vidro. Para a realização desse trabalho, os corpos de prova foram envelhecidos termicamente por um período de $700{ }^{\circ} \mathrm{C}$ por um período de $250 \mathrm{~h}$. Nesta pesquisa o material foi submetido ao ensaio de flexão em quatro 
pontos para a determinação do seu desempenho mecânico; e analisado microscopicamente fazendo o uso da microscopia eletrônica com o intuito de se caracterizar as amostras e o comportamento destas frente às várias condições de carregamento térmico. Observou com isso, que o módulo de elasticidade à flexão diminuiu, enquanto o atrito interno aumentou com os números crescentes de ciclos térmicos. A degradação do material foi atribuída à viscosidade da matriz; e à oxidação das fibras ocorreu devido às exposições prolongadas nas altas temperaturas. Já os danos micro estruturais observados foram à formação de porosidade dentro da matriz e na interface fibra/matriz

\section{MATERIAIS E MÉTODOS}

A metodologia aplicada na pesquisa teve início na determinação e especificação das matérias-primas, seguida da confecção do compósito, realização dos ensaios para caracterização e verificação das propriedades mecânicas usando as normas mencionadas anteriormente, por fim, foi realizado o estudo da fratura final do material, como simplificado e mostrado no fluxograma abaixo.

Figura 1: Fluxograma da metodologia



\subsection{Matérias-Primas}

A matriz usada no trabalho foi à resina polimérica termofixa poliéster insaturada do tipo orto-teraftálica com especificação técnica da Novapol - L20, cujas especificações técnicas, fornecidas pelo fabricante acima mencionado, são mostrados na Tabela 1.

Tabela 1: Especificação da Resina Poliéster. Fonte: Novapol L-120

\begin{tabular}{c|c}
\hline Descrição & $\begin{array}{c}\text { Especificação } \\
\text { Técnica }\end{array}$ \\
\hline
\end{tabular}




\begin{tabular}{c|c}
\hline Viscosidade Brook (SPD & $(250-350) \mathrm{cP}$ \\
2/60rpm) & \\
Densidade $\left(25^{\circ} \mathrm{C}\right)$ & $1,23 \mathrm{~g} / \mathrm{m}^{3}$ \\
Gel Time $\left(1 \% \mathrm{MEKP}-25^{\circ} \mathrm{C}\right)$ & $(9-13) \mathrm{min}$ \\
Pico Exotérmico & $(150-190)^{\circ} \mathrm{C}$ \\
Índice de Acidez & Máximo $30 \mathrm{Mg} \mathrm{KOH} / \mathrm{g}$ \\
\hline
\end{tabular}

A composição química da resina está disposta na tabela 2, essas informações foram retiradas de uma análise através da Espectrometria de Fluorescência de X-Ray. Usando uma corrente entre 5 a $50 \mathrm{kV}$ e uma amperagem entre 1 a 1,000 A.

Tabela 2: Compostos e percentual da composição química da Resina Poliéster. Fonte: Do Autor

\begin{tabular}{c|c}
\hline Composto Químico & $\begin{array}{c}\text { Percentual da } \\
\text { Composição }\end{array}$ \\
\hline $\mathrm{CaO}$ & $59.305 \%$ \\
$\mathrm{SiO}_{2}$ & $12.415 \%$ \\
$\mathrm{Fe}_{2} \mathrm{O}_{3}$ & $10.967 \%$ \\
$\mathrm{Co}_{2} \mathrm{O}_{3}$ & $9.442 \%$ \\
$\mathrm{Al}_{2} \mathrm{O}_{3}$ & $3.597 \%$ \\
$\mathrm{TiO}_{2}$ & $3.113 \%$ \\
$\mathrm{~K}_{2} \mathrm{O}$ & $1.161 \%$ \\
\hline
\end{tabular}

A fibra de vidro-E, aqui usada como material de reforço foi fornecida pela empresa MAXEPOXI, com designação comercial de WR200. Na Figura 2, é possível observar a fibra de vidro na foram de tecido bidirecional e na tabela 3 suas especificações técnicas fornecidas pelo fabricante.

Figura 2: Fibra de vidro-E disposta na forma de tecido bidirecional. Fonte: Do Autor
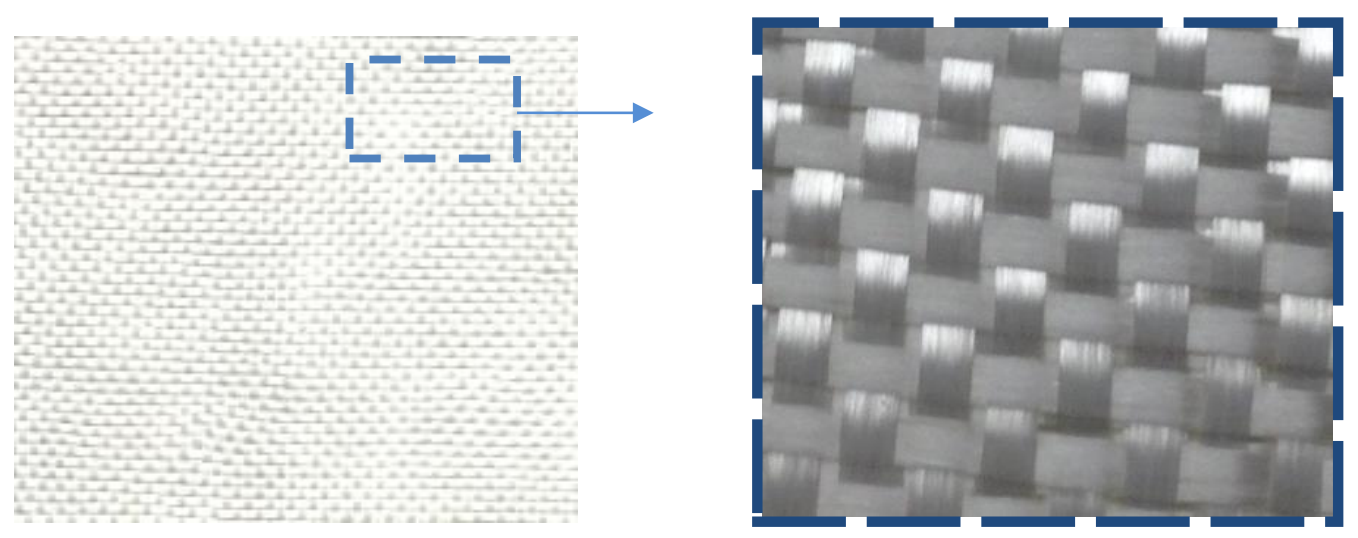
Tabela 3: Especificação dos Tecidos de Fibra de Vidro-E. Fonte: MAXEPOXI

\begin{tabular}{c|c}
\hline Características do Tecido & Fibra de Vidro \\
\hline Gramatura $\left(\mathrm{g} / \mathrm{m}^{2}\right)$ & $200 \pm 20$ \\
Largura $(\mathrm{cm})$ & $130 \pm 3$ \\
Metros Lineares por rolo $(\mathrm{m})$ & $100 \pm 5$ \\
Metro quadrado por rolo $\left(\mathrm{m}^{2}\right)$ & $130 \pm 17$ \\
Espessura $(\mathrm{mm})$ & $0,21 \pm 0,3$ \\
Carga de Ruptura Teórica do Urdume $(\mathrm{Kgf} / \mathrm{cm})$ & $50,0 \pm 10$ \\
Carga de Ruptura Teórica da Trama $(\mathrm{Kgf} / \mathrm{cm})$ & $50,0 \pm 10$ \\
Tipo de Padronagem & Tela \\
Tipo do tecido & Plano \\
\hline
\end{tabular}

\subsection{Confecção dos Compósitos}

O compósito do referido estudo foi confeccionado mediante o processo de laminação manual (Hand-lay-up), sendo feita esta laminação na Tecniplas Indústria e Comercio Ltda. Foi feito apenas um laminado compósito como dito anteriormente, com cinco camadas de reforço e impregnado com a matriz poliéster do tipo teraftálica; para a catalisação fez-se uso do peróxido metil-etil-cetona (MEKP) numa proporção de $1 \%$ do volume de resina, sendo o processo de cura na temperatura ambiente. A disposição das fibras para a obtenção do compósito final pode ser vista na figura 3.

Figura 3: Disposição das fibras

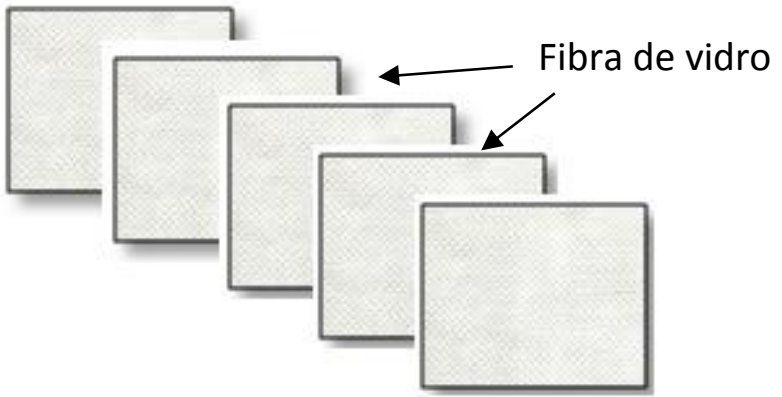

\subsection{Mecanismo de Envelhecimento}

O ensaio de envelhecimento na estufa foi baseado na norma ASTM D 5427 - 2014, esta norma descreve os procedimentos para o envelhecimento acelerado de tecidos de retenção inflável e quando se faz necessário um nível preparatório para outros métodos de ensaio, como por exemplo, ensaio mecânicos. O ensaio de envelhecimento foi realizado utilizando uma estufa marca Nova Ética, modelo 400/2ND, com uma potência de 700W. Como dito anteriormente, foram realizados ciclos de envelhecimento intermitentes e alternados. Primeiro, foi realizado o envelhecimento com umidade; em seguida, o envelhecimento com calor. Foram condicionados 8 corpos de prova. O ensaio teve duração de 672 horas (28 dias). Os corpos de prova eram mudados de posição a cada 7 dias de exposição para obter uma uniformidade no envelhecimento. 


\subsection{Ensaio Mecânico}

O ensaio mecânico de tração uniaxial foi realizado pela máquina "SHIMADZU", modelo AGX 300 com uma capacidade máxima de 30 toneladas e em temperatura ambiente $\left( \pm 25^{\circ} \mathrm{C}\right)$. Em seguida o corpo de prova foi fixado nas garras da máquina. $O$ ensaio foi realizado a uma velocidade de $1 \mathrm{~mm} / \mathrm{min}$, medindo-se as seguintes propriedades mecânicas: tensão máxima de ruptura ( $\mathrm{MPa}$ ), módulo elástico (GPa) e deformação (\%). Foram ensaiados todos os corpos de prova para as diferentes condições: estado seco, imerso em petróleo e imerso em petróleo com envelhecimento. Os corpos de provas foram cortados de acordo com a norma ASTM D790 e as dimensões podem ser observadas na Figura 4.

Figura 4: Corpo de prova para ensaio de tração

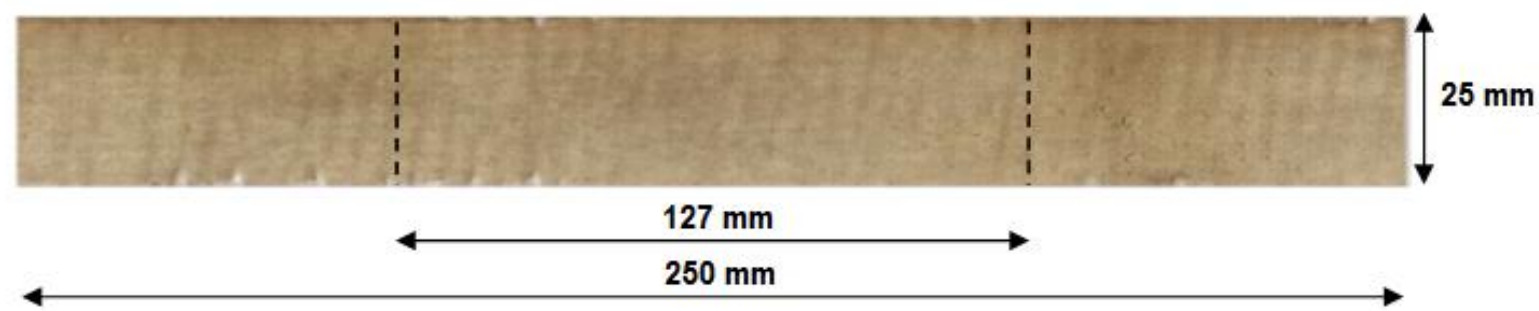

\subsection{Caracterização da Fratura Final}

A caracterização da fratura final dos corpos de provas submetidos aos ensaios mecânicos e as condições impostas teve em seu primeiro plano, detectar possíveis irregularidades no processo de fabricação e, posteriormente, verificar a presença de algum desgaste superficial dos mesmos antes do processo de imersão e envelhecimento. Processo fundamental já que o envelhecimento altera o comportamento estrutural dos compósitos. Para isso foi utilizado o microscópio eletrônico de varredura, da marca Hitachi, modelo TM 3000, utilizou-se uma tensão de $15 \mathrm{kV}$, sendo o material disposto na porta amostra, com o auxílio de uma fita metálica condutora a base de carbono.

\section{RESULTADOS E DISCUSSÕES}

\subsection{Ensaio de Tração}

Na Figura 5, podemos ver as curvas tensão versus deformação, obtidas no ensaio de tração uniaxial, podemos observar que para as três condições, o material obteve o mesmo comportamento mecânico, isso se deu de forma linear até a porção elástica, em seguida, entrou na parte plástica até a ruptura final do mesmo. 
Figura 5: Curvas Tensão X Deformação para o ensaio de tração
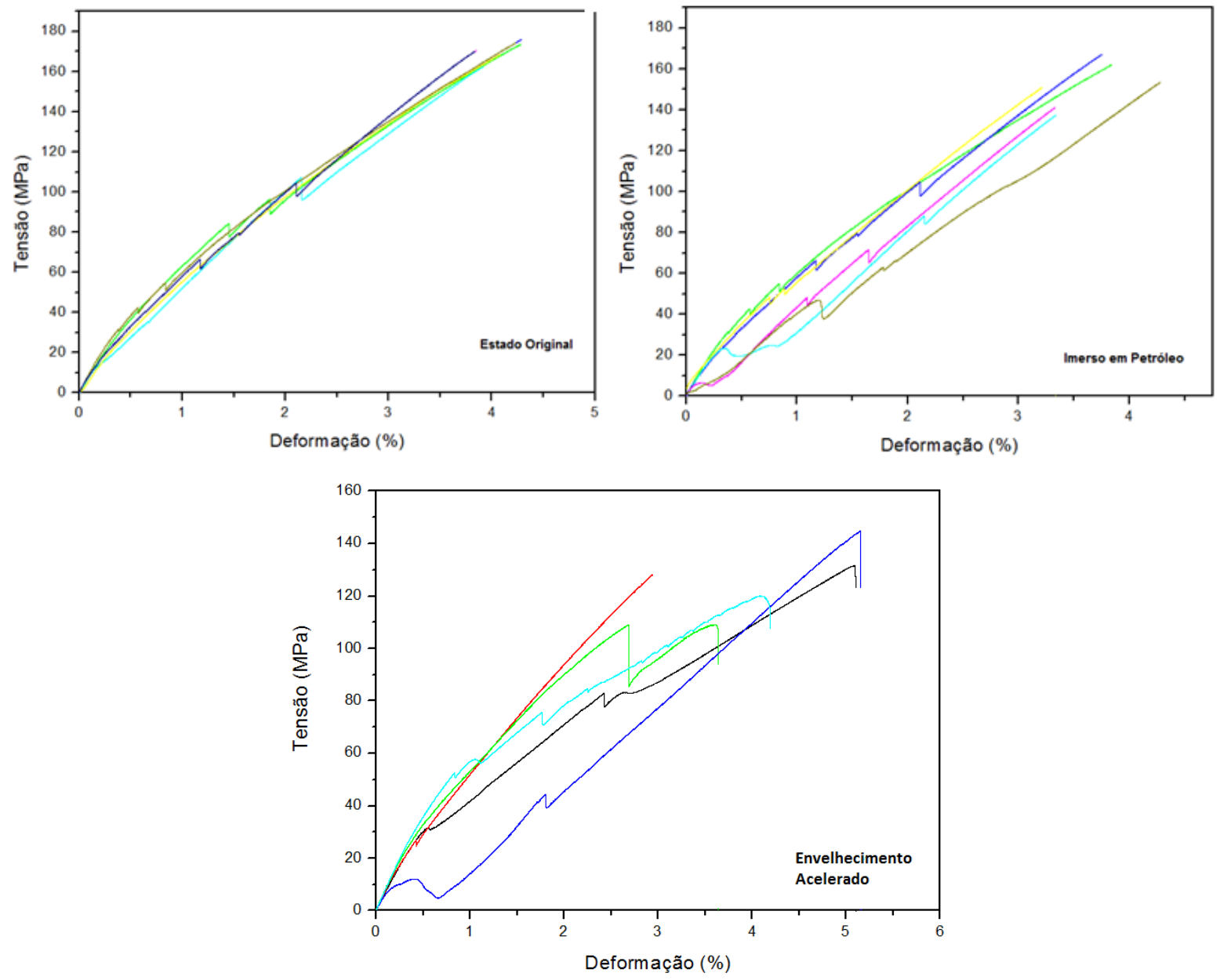

Na tabela 4, as propriedades mecânicas são mostradas com os seus respectivos desviospadrão, lembrando que para efeitos da estatística de resultados foram ensaiados oito CP's para cada condição. Vemos que o PRFV imerso em petróleo (PRFV/IP) apresentou uma queda de $7,52 \%$ quando comparado ao estado original para a tensão de máxima, caindo de $170,09 \mathrm{MPa}$ para 157,29 MPa. Para essa mesma propriedade o PRFV submetido ao envelhecimento acelerado (PRFV/EA) também apresentou uma queda de $25,47 \%$ quando comparado ao seco, e de $16,62 \%$ quando comparado ao imerso em petróleo.

O módulo elástico do material apresentou um comportamento decrescente, uma vez que esse decréscimo traz uma queda na rigidez do material para os três condicionamentos. Com isso, podemos dizer que o petróleo e o envelhecimento em umidade e calor interferiram no valor do módulo de elasticidade. Em termos quantitativos a faixa de valores entre o valor máximo e mínimo foi entre 3 a $4 \mathrm{GPa}$. Para a imersão em petróleo e para o envelhecimento, a retenção percentual para o módulo se compararmos ao estado original foi de $16,32 \%$ e $28,96 \%$, respectivamente.

A deformação à ruptura apresentou-se de forma crescente para as condições, nos quais os valores percentuais forma na ordem $4 \%$. Porcentagem essa que a níveis de instabilidade estrutura é quase que desprezível.

Tabela 4: Propriedades Mecânicas para o ensaio de tração 


\begin{tabular}{c|c|c|c} 
Tensão última (MPa) & $170,09 \pm 5,217$ & $157,29 \pm 6,948$ & $126,766 \pm 13,342$ \\
Módulo de elasticidade & $4,171 \pm 1,471$ & $3,49 \pm 3,345$ & $2,963 \pm 6,518$ \\
(GPa) & $4,08 \pm 0,202$ & $4,54 \pm 0,598$ & $4,467 \pm 1,111$ \\
\hline
\end{tabular}

\subsection{Caracterização da Fratura Final}

A Figura 6 traz a caracterização da fratura no PRFV-EO (estado original), esta começou a ser observada durante o carregamento, onde a trama do tecido ficou em evidência, isso foi provocado pela desaderência da resina durante a aplicação da carga, fazendo surgi pontas de fibras. Este fato resulta numa baixa aderência na interface fibra/matriz; e isso provocou fendas e falta de impregnação. Não podemos deixar de destacar a fratura adesiva na fibra, deixando as fibras de vidro limpas, sem nenhum tipo de impregnação.

Figura 6: Microscopia para o PRFV/EO

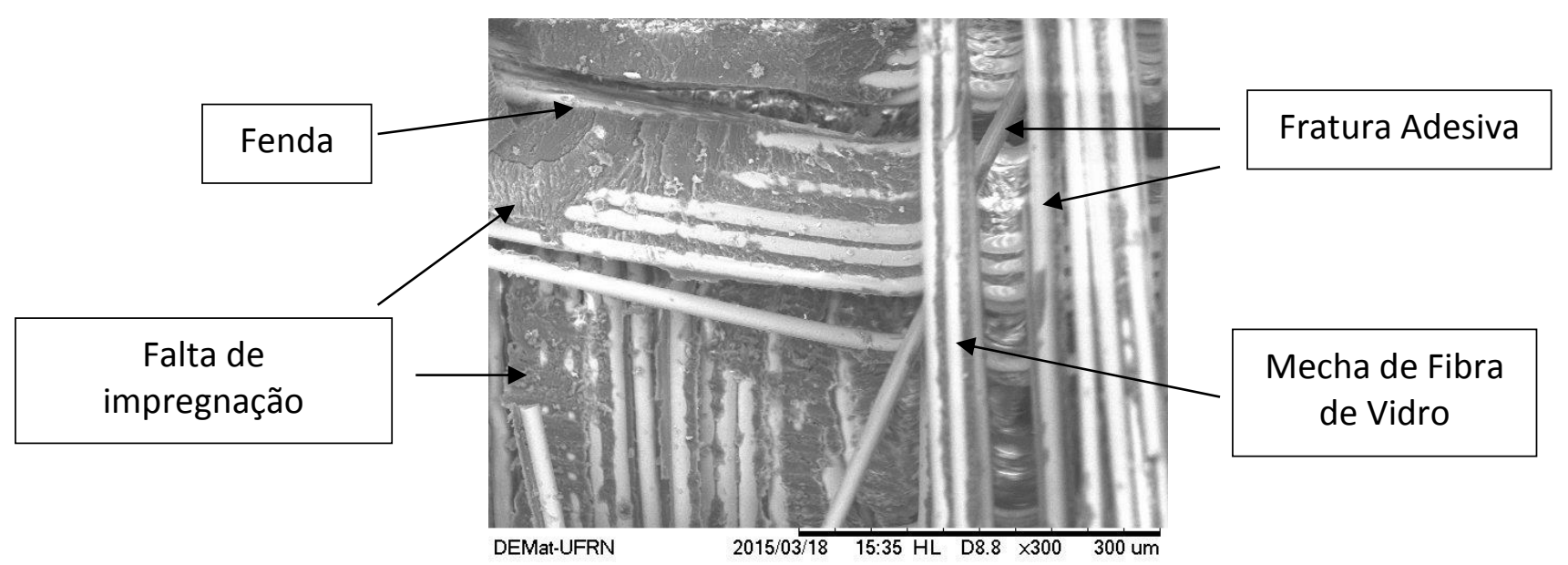

$\mathrm{Na}$ figura 7, podemos ver a fratura final sobre visão microscópica. A presença da impregnação do petróleo próxima a uma região rica em resina pode ser visto também os tipos de fratura adesiva e coesiva na fibra.

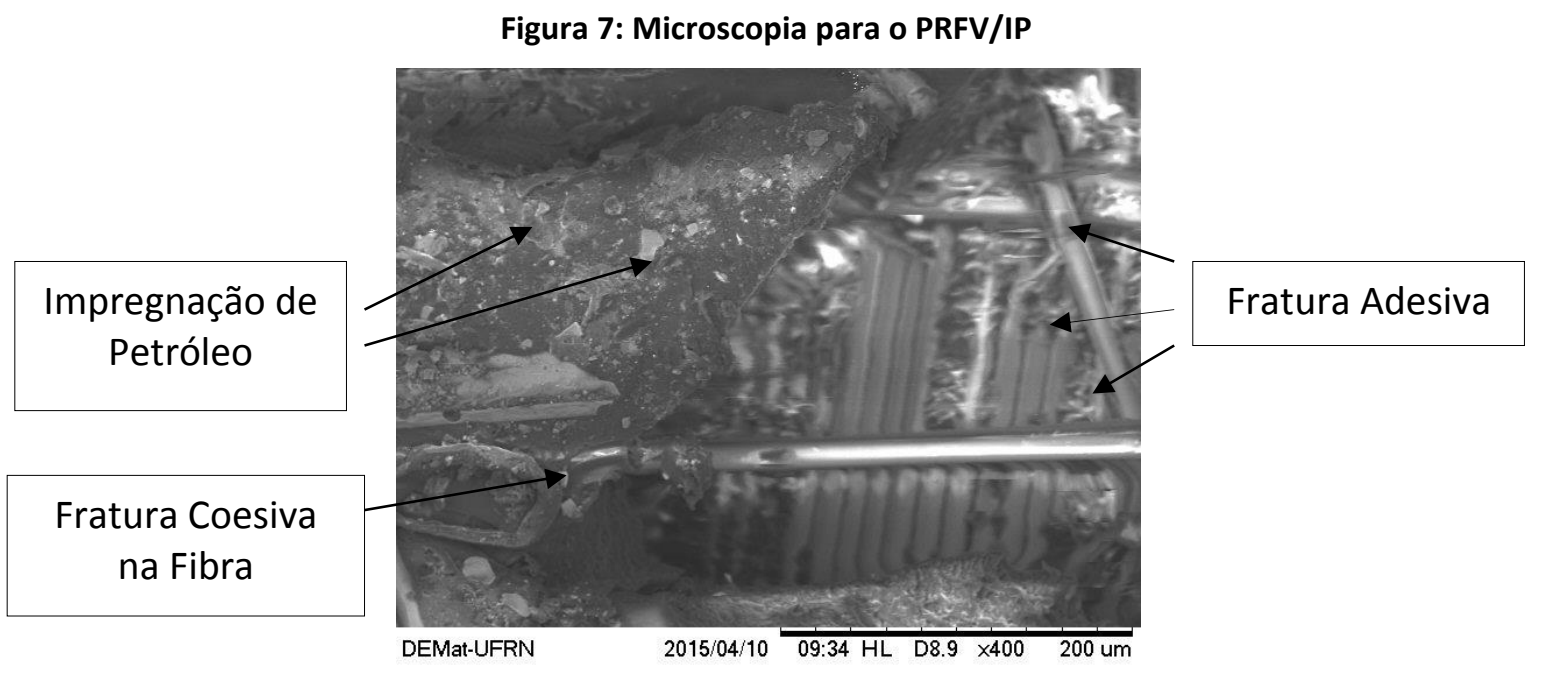


O comportamento da fratura final para o PRFV/EA foi observado a fragmentação da resina ao longo da mecha de fibra de vidro, ficando em evidencia a interface fibra/matriz e a presença de fratura coesiva nas fibras.

Figura 8: Microscopia para o PRFV/EA



\section{CONCLUSÃO}

Ao compararmos o comportamento mecânico do material imerso em petróleo com o estado original, para o ensaio de tração uniaxial, podemos observar que para algumas propriedades mecânicas tiveram uma queda. Ou seja, para a tensão máxima obteve-se uma queda de $8 \%$, para o módulo elástico, obteve-se um percentual de queda de $17 \%$. Já a deformação teve um aumento em torno de $11 \%$.

A comparação do material imerso e submetido ao envelhecimento com o estado original, para o ensaio de tração, obteve novamente uma queda, para esta situação as perdas das propriedades foram mais significativas, para a tensão máxima e módulo de elasticidade, foi de $26 \%$ e $27 \%$, respectivamente. Já para a deformação, o material imerso e envelhecido, um aumento foi observado em $9 \%$.

$\mathrm{Na}$ caracterização da fratura, os danos nos materiais apresentaram as mais diferentes formas, podemos detectar as fraturas adesivas, fraturas coesivas na fibra e na matriz, erosão da resina, interface fibra/matriz, desaderência fibra/matriz, trincas e a impregnação do petróleo.

\section{REFERÊNCIAS}

Albuquerque, J. A. C. O Plástico na Pratica: Manual de Aplicações. Porto Alegre: 2a ed.; Editora Sagra Luzzato, 1999.

ASTM D 3039 - Standard Test Methods for Tensile Properties of Polymer Matrix Composite Materials - American Society for Testing and Materials, 2006.

ASTM D 5427 - Standard Practice for Accelerated Aging of Inflatable Restraint Fabrics American Society for Testing and Materials, 2014.

BARJASTEH, E., BOSZE, E. J., TSAI, Y. I., NUTT, S.R. Thermal aging of fiberglass/carbon-fiber hybrid composites. Elsevier Science Ltda. Part A: Composites, v.49, p.2038-2045, 2009. 
BOCCACCINI, A. R., BOCCACCINI, D. N., PEARCE, D. H., JANCZAK-RUSCH J. et al. Assessment of thermal shock induced damage in silicon carbide fibre reinforced glass matrix composites, Materiales de Construccion, v. 1998, p. 19-35, 1998.

CERNY, I., MAYER, R. M. Fatigue of selected GRP composite components and joints with damage evaluation. Composite Structures, v.94, p.664-670, 2012.

CUNHA P.J.A.; COSTA L.M.; REZENDE C.M. Influência de Diferentes Condições Higrotérmicas na Resistência à Tração de Compósitos de Fibra de Carbono/Epóxi Modificada. Polímeros: Ciência e Tecnologia, v. 16, n³ 3. p. 193-201, 2006.

GAY, D. Matèriaux Composites. Paris: Editions Hermès, 1991.

KHAN, R. A., KHAN, M. A., SULTANA, S., KHAN, M. N., SHUBHRA, Q. T. H., NOOR, F. G. Mechanical, Degradation, and Interfacial Properties of Synthetic Degradable Fiber Reinforced Polypropylene Composites. Journal of Reinforced Plastics and Composites, v. 29, n.3, 2010.

KHAN, R A., KHAN, M. A., ZAMAN, H. U., PERVIN, KHAN, S. N., SULTARA, S., SAHA, M., MUSTAFA, A. I. Comparative Studies of Mechanical and Interfacial Properties Between Jute and E-glass Fiber-reinforced Polypropylene Composites. Journal of Reinforced Plastics and Composites, v.29, n.7, 2010.

HUANG, Y., GE, Z., ZHAO, C., DONG, J., SHITIAN, M., MA, W., WANG, G., QIAN, X. Solvothermal Recovery Of Carbon Fibers From Thermoset Polymer-Based Carbon Fiber Reinforced Polymers. Journal of Reinforced Plastics and Composites. V. 0, p. 1-11. 2015.

LEVY, F. N., PARDINI, L. C. Compósitos Estruturais - Ciência e Tecnologia.1ạ edição. São Paulo: Edgard Blucher, 2006.

MCCONNELL, V. P. Advanced Composites, 4, p. 27, 1990.

MENDONÇA, P.T.R. Materiais Compostos \& Estruturas-Sanduíches. Barueri: Manoele, 2005.

SHAKERI A.; GHASEMIAN A. Water Absorption and Thickness Swelling Behavior of Polypropylene Reinforced with Hybrid Recycled Newspaper and Glass Fiber. Springer Science and Business Media B.V., v.17, p.183-193, 2010.

STRONG, A. B. - Fundamentals of Composites Manufacturing: Materials, Methods, and Applicatinos. SME, Dearbon , 1989.

SU, M., GU, A., LIANG, G., YUAN, L. The effect of oxygen-plasma treatment on Kevlar fibers and the properties of Kevlar fibers/bismaleimide composites. Applied Surface Science, v. 257, p. 3158-3167. 2001.

TAN, S. C. - "Stress Concentrations in Laminated Composites", Technomic Publishing Co., Inc.,Lancaster (1994). 